VOL. 49 (1994) [341-346]

\title{
THE APPROXIMATION PROPERTY AND NILPOTENT IDEALS IN AMENABLE BANACH ALGEBRAS
}

\author{
R.J. LOY AND G.A. WILLIS
}

It is well known that non-zero nilpotent ideals in amenable Banach algebras must be infinite-dimensional. We show that under certain additional hypotheses such ideals cannot even have the approximation property.

In $[4$, Corollary 3.9], it is shown that an amenable Banach algebra cannot have nonzero, finite-dimensional radical. This is because in such algebras, any finite-dimensional nilpotent ideal must be zero; see [7, Proposition VII.2.31] for an alternative proof of the underlying result. The purpose of this note is to show that, in various circumstances, the condition that the ideal be finite-dimensional may be relaxed to the condition that the ideal have the approximation property of Grothendieck.

The approximation property has had other connections with Banach algebra cohomology. It first arose in [9], with the question as to the amenability of $\mathcal{K}(E), E$ a Banach space, and in [13] where it is shown that a biprojective, unital Banach algebra having the approximation property is finite-dimensional and semisimple. Extensive use has since been made in the work of Selivanov, $[10,11]$, in particular extending the result of [13], and, more recently, [12]. The amenability of $\mathcal{K}(E)$ has been investigated in $[6]$ and [5].

We first recall the definition of amenability for Banach algebras. For a Banach algebra, $\mathcal{A}$, the product map extends to a map $\pi: \mathcal{A} \widehat{\otimes} \mathcal{A} \rightarrow \mathcal{A}$, where $\mathcal{A} \widehat{\otimes} \mathcal{A}$ denotes the projective tensor product. The projective tensor product becomes a Banach $\mathcal{A}$-bimodule if we define $a .(b \otimes c)=a b \otimes c$ and $(b \otimes c) . a=b \otimes c a$ and extend these actions to $\mathcal{A} \widehat{\otimes} \mathcal{A}$ by linearity and continuity. Then $\pi$ becomes an $\mathcal{A}$-bimodule homomorphism. An approximate diagonal for $\mathcal{A}$ is a bounded net $\left\{d_{\lambda}\right\}_{\lambda \in \Lambda}$ in $\mathcal{A} \hat{\otimes} \mathcal{A}$ satisfying $\lim _{\lambda}\left\|a \cdot d_{\lambda}-d_{\lambda} \cdot a\right\|=0$ for all $a$ in $\mathcal{A}$ and $\left\{\pi\left(d_{\lambda}\right)\right\}_{\lambda \in \Lambda}$ is a bounded approximate identity for $\mathcal{A}$. Then $\mathcal{A}$ is amenable if it has an approximate diagonal. Note that amenability usually is defined in terms of derivations from $\mathcal{A}$, see [9, Section 5$]$, or $[1$, Definition 43.2]. However, as is shown in [2] and [9], the above condition is equivalent to that definition.

Received 9th June, 1993

Copyright Clearance Centre, Inc. Serial-fee code: 0004-9729/94 \$A2.00+0.00. 
The Banach algebra $\mathcal{A}$ is said to be biprojective if there is a continuous $\mathcal{A}$-bimodule homomorphism, $T: \mathcal{A} \rightarrow \mathcal{A} \widehat{\otimes} \mathcal{A}$, such that $\pi \circ T$ is the identity map on $\mathcal{A}$. If $\mathcal{A}$ is biprojective and has a bounded approximate identity, $\left\{u_{\lambda}\right\}_{\lambda \in \Lambda}$, then $\left\{T\left(u_{\lambda}\right)\right\}_{\lambda \in \Lambda}$ is an approximate diagonal for $\mathcal{A}$ and so $\mathcal{A}$ is amenable. The converse is not true. Biprojectivity, in the presence of a bounded approximate identity, is a much stronger property than amenability, for example, the group algebra $L^{1}(G)$ is amenable if and only if $G$ is amenable but is biprojective if and only if $G$ is compact.

A Banach space $E$ is said to have the approximation property if, for each compact subset $K$ of $E$, and each $\varepsilon>0$, there is a finite rank operator $F$ on $E$ such that $\|F(x)-x\|<\varepsilon$ for each $x$ in $K$. It is well known that if $E$ has the approximation property, and $T: E \rightarrow X$ is an embedding, then for each Banach space $Y$ the map $T \otimes I_{Y}: E \widehat{\otimes} Y \rightarrow X \widehat{\otimes} Y$ is injective. To see this, let $\sum e_{k} \otimes y_{k}$ be an element of $E \hat{\otimes} Y$ which is mapped to zero under $T \otimes I_{Y}$. We may suppose that $e_{k} \rightarrow 0$ and $\sum\left\|y_{k}\right\|<1$. Take $\varepsilon>0$ and a finite rank operator $F$ on $E$ such that $\left\|F\left(e_{k}\right)-e_{k}\right\|<\varepsilon$ for each $k$. Such $F$ may be extended to $F^{\prime}: X \rightarrow E$ with $F=F^{\prime} \circ T$. Then

$$
\begin{aligned}
\left\|\sum e_{k} \otimes y_{k}\right\|_{E \widehat{\otimes} Y} & \leqslant\left\|\sum F\left(e_{k}\right) \otimes y_{k}\right\|_{E \widehat{\otimes} Y}+\varepsilon \\
& \leqslant\left\|\left(F \otimes I_{Y}\right) \sum e_{k} \otimes y_{k}\right\|_{E \widehat{\otimes} Y}+\varepsilon \\
& \leqslant\left\|F^{\prime}\right\|\left\|\left(T \otimes I_{Y}\right) \sum e_{k} \otimes y_{k}\right\|_{X \widehat{\otimes} Y}+\varepsilon \\
& =\varepsilon .
\end{aligned}
$$

It follows that $\sum e_{k} \otimes y_{k}=0$ in $E \widehat{\otimes} Y$.

We now have

Proposition 1. Let $\mathcal{A}$ be a biprojective Banach algebra with a central approximate identity, and let $\mathcal{N}$ be a non-zero nilpotent ideal in $\mathcal{A}$. Then $\mathcal{N}$ does not have the approximation property.

Proof: Since $\mathcal{N}$ is non-zero and nilpotent there is a positive integer $n$ such that $\mathcal{N}^{n} \neq(0)$ but $\mathcal{N}^{n+1}=(0)$. Let $\mathcal{J}$ be the closed linear span of $\mathcal{N}^{n}$, so that $\mathcal{J}$ is not zero. Let $T: \mathcal{A} \rightarrow \mathcal{A} \widehat{\otimes} \mathcal{A}$ be a continuous $\mathcal{A}$-bimodule homomorphism such that $\pi \circ T$ is the identity map on $\mathcal{A}$.

Let $J: \mathcal{N} \rightarrow \mathcal{A}$ be the inclusion map, $Q: \mathcal{A} \rightarrow \mathcal{A} / \mathcal{J}$ be the quotient map and $\tilde{\pi}: \mathcal{N} \hat{\otimes} \mathcal{A} \rightarrow \mathcal{N}$ be the map induced by the product on $\mathcal{A}$. Note that $J \circ \tilde{\pi}=\pi \circ\left(J \otimes I_{\mathcal{A}}\right)$, where $I_{\mathcal{A}}$ denotes the identity map on $\mathcal{A}$. Since $\mathcal{N} \mathcal{J}=(0), \tilde{\pi}$ factors through $\mathcal{N} \hat{\otimes} \mathcal{A} / J$ as $\tilde{\pi}=P \circ\left(I_{\mathcal{N}} \otimes Q\right)$, where $P: \mathcal{N} \widehat{\otimes} \mathcal{A} / \mathcal{J} \rightarrow \mathcal{N}$ is the map induced by the action $b \otimes(a+\mathcal{J}) \rightarrow b a$.

Now let $b$ be in $\mathcal{N}$ and let $L_{b}, R_{b}: \mathcal{A} \rightarrow \mathcal{N}$ be the maps of left, respectively right, multiplication by $b$. Then $\tilde{\pi} \circ\left(L_{b} \otimes I_{\mathcal{A}}\right)=L_{b} \circ \pi$. Let $\left\{u_{\lambda}\right\}_{\lambda \in \Lambda}$ be a central 
approximate identity for $\mathcal{A}$ and put $v_{\lambda}=\left(\left(I_{\mathcal{N}} \otimes Q\right) \circ\left(L_{b} \otimes I_{\mathcal{A}}\right) \circ T\right)\left(u_{\lambda}\right)$ which belongs to $\mathcal{N} \widehat{\otimes} \mathcal{A} / \mathcal{J}$. Then $P\left(v_{\lambda}\right)=\left(\tilde{\pi} \circ\left(L_{b} \otimes I_{\mathcal{A}}\right) \circ T\right)\left(u_{\lambda}\right)=\left(L_{b} \circ \pi \circ T\right)\left(u_{\lambda}\right)=b u_{\lambda}$, which converges to $b$. Hence, if $b$ is not zero, then $v_{\lambda}$ is not zero for some $\lambda$. Now choose a fixed and non-zero $b$ in $\mathcal{J}$, and a fixed $\lambda$ such that $v_{\lambda}$ is not zero.

Since $u_{\lambda}$ is central and $T$ is a bimodule homomorphism, b.T $\left(u_{\lambda}\right)=T\left(b u_{\lambda}\right)=$ $T\left(u_{\lambda}\right) . b$ in $\mathcal{A} \widehat{\otimes} \mathcal{A}$. It follows that

$$
\left(\left(J \otimes I_{\mathcal{A}}\right) \circ\left(L_{b} \otimes I_{\mathcal{A}}\right) \circ T\right)\left(u_{\lambda}\right)=\left(\left(I_{\mathcal{A}} \otimes J\right) \circ\left(I_{\mathcal{A}} \otimes R_{b}\right) \circ T\right)\left(u_{\lambda}\right)
$$

Hence,

$$
\begin{aligned}
\left(J \otimes I_{\mathcal{A} / \mathcal{J}}\right)\left(v_{\lambda}\right) & =\left(\left(J \otimes I_{\mathcal{A} / \mathcal{J}}\right) \circ\left(I_{\mathcal{N}} \otimes Q\right) \circ\left(L_{b} \otimes I_{\mathcal{A}}\right) \circ T\right)\left(u_{\lambda}\right) \\
& =\left(\left(I_{\mathcal{A}} \otimes Q\right) \circ\left(J \otimes I_{\mathcal{A}}\right) \circ\left(L_{b} \otimes I_{\mathcal{A}}\right) \circ T\right)\left(u_{\lambda}\right) \\
& =\left(\left(I_{\mathcal{A}} \otimes Q\right) \circ\left(I_{\mathcal{A}} \otimes J\right) \circ\left(I_{\mathcal{A}} \otimes R_{b}\right) \circ T\right)\left(u_{\lambda}\right) \\
& =\left(\left(I_{\mathcal{A}} \otimes\left(Q \circ J \circ R_{b}\right)\right) \circ T\right)\left(u_{\lambda}\right) \\
& =0
\end{aligned}
$$

because $Q \circ J \circ R_{b}=0$. Since $v_{\lambda}$ is not zero, it follows that $J \otimes I_{\mathcal{A} / \mathcal{J}}: \mathcal{N} \widehat{\otimes} \mathcal{A} / \mathcal{J} \rightarrow$ $\mathcal{A} \widehat{\otimes} \mathcal{A} / \mathcal{J}$ has non-zero kernel. Therefore, $\mathcal{N}$ does not have the approximation property.

REMARK 1. The hypotheses can be weakened, to require that for some non-zero $b$ in $\mathcal{J}$, there be a sequence $\left\{u_{n}\right\}_{n \geqslant 1}$ such that

$$
u_{n} b=b u_{n} \rightarrow b
$$

Further, the condition that $\mathcal{N}$ be nilpotent can also be weakened, to require that the intersection of $\mathcal{N}$ with its right annihilator be non-zero.

REMARK 2. It was pointed out to the authors by Alexander Khelemskii that some commutativity hypothesis such as (1) is necessary. The algebra of $2 \times 2$ matrices with zero first column is finite-dimensional, biprojective, and has a non-zero nilpotent ideal.

Proposition 2. Let $\mathcal{A}$ be an amenable Banach algebra and suppose that $\mathcal{N}$ is an ideal in $\mathcal{A}$ which is nilpotent, has the approximation property, and is such that multiplication on the left by any element of $\mathcal{N}$ is a compact operator on $\mathcal{A}$. Then $\mathcal{N}=(0)$.

Proof: As before, suppose that $\mathcal{N}^{n} \neq(0), \mathcal{N}^{n+1}=(0)$, let $\mathcal{J}$ be the closed linear span of $\mathcal{N}^{n}$ and choose a non-zero $b$ in $\mathcal{J}$. We shall derive a contradiction. Also as before, let $J: \mathcal{N} \rightarrow \mathcal{A}$ be the inclusion map, $Q: \mathcal{A} \rightarrow \mathcal{A} / \mathcal{J}$ be the quotient map 
and $\tilde{\pi}: \mathcal{N} \widehat{\otimes} \mathcal{A} \rightarrow \mathcal{N}$ be the map induced by the product on $\mathcal{A}$. We still have that $\tilde{\pi}$ factors through $\mathcal{N} \widehat{\otimes} \mathcal{A} / \mathcal{J}$ as $\tilde{\pi}=P \circ\left(I_{\mathcal{N}} \otimes Q\right)$, where $P: \mathcal{N} \widehat{\otimes} \mathcal{A} / \mathcal{J} \rightarrow \mathcal{N}$.

Let $\left\{d_{\lambda}\right\}_{\lambda \in \Lambda}$ be an approximate diagonal for $\mathcal{A}$, where $d_{\lambda}=\sum_{k=1}^{\infty} u_{k, \lambda} \otimes v_{k, \lambda}$ and we may suppose that there is a constant $M$ such that $\left\|u_{k, \lambda}\right\|<M$ for each $k$ and $\lambda$ and $\sum_{k=1}^{\infty}\left\|v_{k, \lambda}\right\|=1$ for each $\lambda$. Then $\sum_{k=1}^{\infty} b u_{k, \lambda} \otimes Q\left(v_{k, \lambda}\right)$ is in $\mathcal{N} \widehat{\otimes} \mathcal{A} / \mathcal{J}$ and $P\left(\sum_{k=1}^{\infty} b u_{k, \lambda} \otimes\right.$ $\left.Q\left(v_{k, \lambda}\right)\right)=b \pi\left(d_{\lambda}\right)$, which converges to $b$. Since $b$ is non-zero we may suppose, by scaling $b$ if necessary, that

$$
\left\|\sum_{k=1}^{\infty} b u_{k, \lambda} \otimes Q\left(v_{k, \lambda}\right)\right\|_{\mathcal{N} \otimes \mathcal{A} / \mathcal{J}} \geqslant 1
$$

for all $\lambda$ sufficiently large.

On the other hand, since $\lim _{\lambda}\left\|b . d_{\lambda}-d_{\lambda} . b\right\|=0$ and $d_{\lambda} . b$ belongs to $\mathcal{A} \widehat{\otimes} \mathcal{J}$, we have that

$$
\left\|\sum_{k=1}^{\infty} b u_{k, \lambda} \otimes Q\left(v_{k, \lambda}\right)\right\|_{\mathcal{A} \widehat{\otimes} \mathcal{A} / \mathcal{J}}=\left\|\left(I_{\mathcal{A}} \otimes Q\right)\left(\sum_{k=1}^{\infty} b u_{k, \lambda} \otimes v_{k, \lambda}\right)\right\|_{\mathcal{A} \widehat{\otimes} \mathcal{A} / \mathcal{J}} \rightarrow 0
$$

Since the map $u \mapsto b u$ is a compact operator, $\left\{b u_{k, \lambda}: k=1,2,3, \ldots ; \lambda \in \Lambda\right\}^{-}$is a compact set. Hence, since $\mathcal{N}$ has the approximation property, there is finite rank operator $F: \mathcal{A} \rightarrow \mathcal{N}$ such that $\left\|b u_{k, \lambda}-F\left(b u_{k, \lambda}\right)\right\|<1 / 2$ for each $k$ and $\lambda$. It follows that

$$
\begin{aligned}
\left\|\sum_{k=1}^{\infty} b u_{k, \lambda} \otimes Q\left(v_{k, \lambda}\right)\right\|_{\mathcal{N} \hat{\mathcal{A}} / \mathcal{J}} & \leqslant\left\|\sum_{k=1}^{\infty}\left(b u_{k, \lambda}-F\left(b u_{k, \lambda}\right)\right) \otimes Q\left(v_{k, \lambda}\right)\right\|_{\mathcal{N} \hat{\otimes} \mathcal{A} \mathcal{J}} \\
& +\left\|\sum_{k=1}^{\infty} F\left(b u_{k, \lambda}\right) \otimes Q\left(v_{k, \lambda}\right)\right\|_{\mathcal{N} \mathcal{A} / \mathcal{J}} \\
& \frac{1}{2}+\left\|\left(F \otimes I_{\mathcal{A} / \mathcal{J}}\right)\left(\sum_{k=1}^{\infty} b u_{k, \lambda} \otimes Q\left(v_{k, \lambda}\right)\right)\right\|_{\mathcal{N} \hat{\otimes} \mathcal{A} \mathcal{J}} \\
\leqslant & \frac{1}{2}+\|F\|\left\|\sum_{k=1}^{\infty} b u_{k, \lambda} \otimes Q\left(v_{k, \lambda}\right)\right\|_{\mathcal{A} \widehat{\mathcal{A}} / \mathcal{J}} \\
& \frac{1}{2}
\end{aligned}
$$

as $\lambda$ increases, contradicting (2). 
It is an open question whether there is a radical, amenable Banach algebra, see the remarks following Corollary 3.9 in [4]. One way to try to construct such an algebra would be to describe it as a direct limit of nilpotent algebras. In a commutative algebra each nilpotent element generates a nilpotent ideal. Hence, by Proposition 1, if we wish to construct a biprojective algebra in this way, then we must construct Banach spaces without the approximation property along the way. By Proposition 2, any such construction of an amenable algebra must produce either an example in which multiplication is not compact, or examples of spaces without the approximation property.

There are commutative amenable algebras which contain nilpotent ideals. They can arise as quotients of commutative group algebras by ideals for which spectral synthesis fails, for example see [14]. It easily follows from a result of Helson [8] that such nilpotent ideals cannot be finite-dimensional. However, the above propositions do not seem to apply to these algebras because they are not biprojective and, generally, multiplication by an element is not a compact operator. We do not know whether multiplication by a nilpotent element can be compact in this situation; if so, then we shall have produced some new examples of Banach spaces without the approximation property.

It is not clear whether the biprojectivity condition in Proposition 1 or the compactness condition in Proposition 2 is necessary. Perhaps it is true in general that non-zero nilpotent ideals in amenable Banach algebras cannot have the approximation property. More specifically, we conjecture that if $\mathcal{J} \subset \mathcal{I}$ are two distinct ideals in a commutative group agebra $\mathcal{A}(G)$ which have the same hull, then $\mathcal{I} / \mathcal{J}$ does not have the approximation property. It is also unknown whether $\mathcal{I} / \mathcal{J}$ can have a Banach space complement in $\mathcal{A}(G) / \mathcal{J}$, though this certainly fails if $(\mathcal{I} / \mathcal{J})^{2} \neq \mathcal{I} / \mathcal{J}$, see [3], and certainly, [1, Theorem 4.3] shows that $\mathcal{I} / \mathcal{J}$ cannot have an algebra complement in $\mathcal{A}(G) / \mathcal{J}$. Since $\mathcal{A}(G)$ always has the approximation property, it is thus of interest to determine whether $\mathcal{A}(G) / \mathcal{J}$ has the approximation property.

\section{REFERENCES}

[1] W.G. Bade and H.G. Dales, 'The Wedderburn decomposability of some commutative Banach algeras', J. Funct. Anal. 107 (1992), 105-121.

[2] F.F. Bonsall and J. Duncan, Complete normed algebras (Springer-Verlag, Berlin, Heidelberg, New York, 1973).

[3] P.C. Curtis, Jr., 'Complementation problems concerning the radical of a commutative amenable Banach algebra', Proc. Centre Math. Anal. Austral. Nat. Univ. 21 (1989), 56-60.

[4] P.C. Curtis, Jr. and R.J. Loy, 'The structure of amenable Banach algebras', J. London Math. Soc. (2) 40 (1989), 89-104.

[5] N. Grønbæk, B.E. Johnson and G.A. Willis, 'Amenability of Banach algebras of compact operators', preprint (1992). 
[6] N. Grønbæk and G.A. Willis, 'Approximate identities in Banach algebras of compact operators', Canad. Math. Bull. 36 (1993), 45-53.

[7] A.Ya. Helemskii, The homology of Banach and topological algebras (Kluwer, Dordrecht, 1986).

[8] H. Helson, 'On the ideal structure of group algebras', Ark. Mat. 2 (1952), 83-86.

[9] B.E. Johnson, 'Cohomology in Banach algebras', Mem. Amer. Math. Soc. No. 127 (1972), 1-96.

[10] Y.V. Selivanov, 'Projectivity of certain Banach modules and the structure of Banach algebras', Soviet Math. (Iz. VUZ) 22 (1978), 88-93.

[11] Y.V. Selivanov, 'Biprojective Banach algebras', Izv. Akad. Nauk SSSR Ser. Matem. 43 (1979), 1159-1174.

[12] Y.V. Selivanov, 'Homological characterizations of the approximation property for Banach spaces', Glasgow Math. J. 34 (1992), 229-239.

[13] J.L. Taylor, 'Homology and cohomology for topological algebras', Adv. in Math. 9 (1972), 137-182.

[14] N.Th. Varopoulos, 'Spectral synthesis on spheres', Proc. Camb. Philos. Soc. 62 (1966), 379-387.

Department of Mathematics School of Mathematical Sciences Australian National University Australian Capital Territory 0200

\author{
Department of Mathematics \\ The University of Newcastle \\ New South Wales 2308 \\ Australia
}

